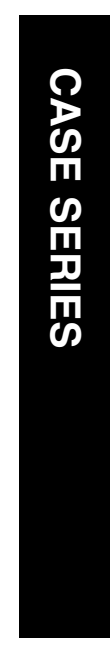

\title{
Medically unexplained visual symptoms in children and adolescents: an indicator of abuse or adversity?
}

${ }^{1}$ Newcastle University, Newcastle Upon Tyne, UK

\section{${ }^{2}$ Department of}

Ophthalmology, Newcastle Upon Tyne Hospitals NHS Foundation Trust, Newcastle Upon Tyne, UK

${ }^{3}$ Institute of Neuroscience, Newcastle University, Newcastle Upon Tyne, UK

Correspondence: MP Clarke, Department of Ophthalmology, Royal Victoria Infirmary, Queen Victoria Rd, Newcastle Upon Tyne, Tyne and Wear, NE1 4LP, UK

Tel: + 019128240 02;

Fax + 01912825446 .

E-mail:

m.p.clarke@ncl.ac.uk

Received: 24 June 2009 Accepted: 24 June 2009 Published online: 31 July 2009

\begin{abstract}
Purpose To highlight abuse and adversity in childhood as an association of medically unexplained symptoms.

Methods Case series.

Results Two young adults and one child (all female) are described with medically unexplained impaired vision and diplopia, which on further investigation were found to be associated with abuse or adversity, and in one case was followed by the development of further unexplained symptoms.

Conclusions Ophthalmologists dealing with children and young people need to ensure that child abuse and adversity are considered as potential underlying associations when dealing with patients with medically unexplained symptoms.
\end{abstract}

Eye (2009) 23, 1866-1867; doi:10.1038/eye.2009.197; published online 31 July 2009

Keywords: Somatiform disorder; medically unexplained visual loss

\section{Introduction}

Medically unexplained symptoms (MUS) remain a frequent cause of presentation to health services. ${ }^{1}$ MUS often elaborate symptoms clearly attributable to physical pathology, ${ }^{2}$ but are often also a manifestation of psychological distress (termed dissociative or Conversion disorders if short lived, and Somatoform disorders if persistent). Patients with MUS are frequently subjected to inappropriate investigations and treatments, which consume significant healthcare resources. The clear link between childhood abuse, neglect and adversity, and presentation in adult life with $\mathrm{MUS}^{3}$ has proved more difficult to show in children. ${ }^{4}$ There is only one recent review of MUS in adults presenting with visual disorders, ${ }^{2}$ and no recent literature describing the characteristics of medically unexplained visual symptoms in children and young adults. In this paper, we present the cases of a child and two young adults with medically unexplained visual symptoms. In all the three cases, there were significant adverse psychosocial factors, and in one case there was a disclosure of physical abuse.

\section{Case 1}

A 17-year-old girl, with a history of four previous surgical procedures for infantile esotropia, presented with increasingly frequent episodes of vertical diplopia, which was treated with monocular occlusion by her optometrist. This was followed by progressive bilateral loss of visual acuity, causing difficulty while navigating and reading. Assessment of visual acuity was difficult due to photophobia, and variable results were obtained. Apart from a small residual convergent strabismus, ophthalmic examination, and, subsequently, neurological evaluation was normal. Psychiatric evaluation was suggested, but was declined. A history of stressful family relationships (a brother with autism and recent parental divorce) was elicited from the family doctor.

\section{Case 2}

A seventeen-year-old girl presented with an inconsistent 2-year history of intermittent blurring of vision and diplopia. Initial examination had shown a marked convergent 
strabismus and reduced visual acuity (6/36 right; 6/60 left). Despite correction of a small refractive error, acuity remained reduced at 6/18 in each eye, and intermittent diplopia persisted. MR imaging of brain and orbits was normal. Further questioning revealed little evidence of functional disability at home due to reduced acuity, and performance at school had not deteriorated. Stereoacuity was measured as $120 \mathrm{~s}$ of arc. A psychological basis for the symptoms was suggested to the family. Nine months later, the patient presented with a suspected functional neurological disorder with the feet held in equinus, necessitating the use of a wheelchair. A psychiatric evaluation revealed a history of panic and anxiety in stressful situations and a referral was made to clinical psychology services.

\section{Case 3}

A 10-year-old girl presented with a 4-months history of transient horizontal diplopia with headaches. Shortly before presentation, her visual acuity had deteriorated to 6/12 right and 6/18 left. Apart from a small latent convergent strabismus, no abnormality was found on ophthalmic examination. Subsequent enquiry revealed a history of nocturnal episodes of screaming associated with a normal EEG. A disclosure of physical abuse was subsequently made.

\section{Discussion}

MUS may be manifestations of a physical diagnosis, which has not yet been made, of an underlying psychological disorder (including Munchausen syndrome), of deliberate fabrication (malingering), or may have no identifiable association. Medically unexplained visual symptoms in adults are well described. $^{2}$

In children (and adults), MUS may be associated with childhood adversity, neglect and abuse, particularly sexual abuse ${ }^{5}$ as in one of our cases. It is for this reason that it is particularly important that MUS are positively identified so that causes of the underlying psychological distress can be considered.
The management of somatisation is difficult. Sufferers are often resistant to the suggestion that their symptoms may have a psychological basis. Cognitive behaviour therapy and antidepressants may be helpful. ${ }^{6}$

The presentation of Somatoform disorders in paediatric ophthalmology is often dramatic, generating much anxiety. We recommend that clinicians dealing with children with MUS are aware that somatoform disorders can be the cause, and explore the child's social circumstances and initiate appropriate referral if there are concerns about abuse.

\section{Conflict of interest}

The authors declare no conflict of interest.

\section{Summary}

\section{What was known before}

Medically unexplained symptoms may be a symptom of psychological distress and current or previous child abuse.

What this study adds

Presentation with medically unexplained visual symptoms has not been recently described as a manifestation of child abuse/adversity.

\section{References}

1 Stone J, Hewett R, Carson A, Warlow C, Sharpe M. The 'disappearance' of hysteria: historical mystery or illusion? J R Soc Med 2008; 101: 12-18.

2 Griffiths P, Eddyshaw D. Medically unexplained visual loss in adult patients. Eye 2004; 18: 917-922.

3 Barsky AJ, Wool C, Barnett MC, Cleary PD. Histories of childhood trauma in adult hypochondriacal patients. Am J Psychiatry 1994; 151(3): 397-401.

4 Eminson M. Medically unexplained symptoms in children and adolescents. Clin Psychol Rev 2007; 27(7): 855-871.

5 Fiddler M, Jackson J, Kapur N, Wells A, Creed F. Childhood adversity and frequent medical consultations. Gen Hosp Psychiatry 2004; 26(5): 367-377.

6 Burton C. Beyond somatisation: a review of the understanding and treatment of medically unexplained physical symptoms (MUPS). Br J Gen Pract 2003; 53(488): 231-239. 